International Journal of Pure and Applied Mathematics

Volume 90 No. 1 2014, 35-41

ISSN: 1311-8080 (printed version); ISSN: 1314-3395 (on-line version)

url: http://www.ijpam.eu

doi: http://dx.doi.org/10.12732/ijpam.v90i1.5

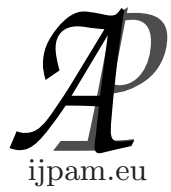

\title{
ON PARA KENMOTSU MANIFOLD
}

\author{
K.L. Sai Prasad ${ }^{1 \S}$, T. Satyanarayana ${ }^{2}$ \\ ${ }^{1}$ Department of Mathematics \\ Gayatri Vidya Parishad College of Engineering for Women \\ Madhurawada, Visakhapatnam, 530 048, INDIA \\ ${ }^{2}$ Department of Mathematics \\ Pragati Engineering College, Surampalem, Near Peddapuram \\ Andhra Pradesh, INDIA
}

\begin{abstract}
A type of para Kenmotsu (briefly $p$-Kenmotsu) manifold in which $R(\xi, X) . C=0$ has been considered, where $C$ is the conformal curvature tensor of the manifold and $R$ is the curvature transformation. It has been shown that such a manifold is conformally flat and hence is an $s p$-Kenmotsu manifold.
\end{abstract}

AMS Subject Classification: 53C15

Key Words: para contact metric manifold, curvature tensor, Ricci tensor, tangent vector

\section{Introduction}

Sato [1] defined the notions of an almost para contact Riemannian manifold. After that, T. Adati and K. Matsumoto [2] defined and studied para-Sasakian and SP-Sasakian manifolds which are regarded as a special kind of an almost contact Riemannian manifolds. Before Sato, Kenmotsu [3] defined a class of almost contact Riemannian manifolds. In 1995, B. B. Sinha and K. L. Sai Prasad [4] have defined a class of almost para contact metric manifolds namely

Received: September 11, 2013

(C) 2014 Academic Publications, Ltd.

$\S_{\text {Correspondence author }}$ url: www.acadpubl.eu 
para Kenmotsu (briefly $p$-Kenmotsu) and special para Kenmotsu (briefly $s p$ -Kenmotsu) manifolds. In a recent paper, the authors Satyanarayana and Sai Prasad [11] have proved that if in a $p$-Kenmotsu manifold $\left(M_{n}, g\right)(n>3)$ the relation $R(X, Y) . C=0$ holds, where $C$ is the conformal curvature tensor of the manifold and $R$ is the Riemannian curvature and $R(X, Y)$ is considered as a derivation of the tensor algebra at each point of the manifold for tangent vectors $X$ and $Y$, then the manifold is conformally flat and hence is an $s p$-Kenmotsu manifold. In this paper, we have generalized this result by taking the weaker hypothesis $R(\xi, X) . C=0$ instead of $R(X, Y) . C=0$ in a $p$-Kenmotsu manifold.

Let $M_{n}$ be an $n$-dimensional differentiable manifold equipped with structure tensors $(\Phi, \xi, \eta)$ where $\Phi$ is a tensor of type $(1,1), \xi$ is a vector field, $\eta$ is a 1-form such that

$$
\begin{gathered}
\eta(\xi)=1 \\
\Phi^{2}(X)=X-\eta(X) \xi ; \bar{X}=\Phi X
\end{gathered}
$$

Then $M_{n}$ is called an almost para contact manifold.

Let ' $g$ ' be the Riemannian metric satisfying such that, for all vector fields $X$ and $Y$ on $M$,

$$
\begin{gathered}
g(X, \xi)=\eta(X) \\
\Phi \xi=0, \eta(\Phi X)=0, \operatorname{rank} \Phi=n-1 \\
g(\Phi X, \Phi Y)=g(X, Y)-\eta(X) \eta(Y)
\end{gathered}
$$

Then the manifold $M n[1]$ is said to admit an almost para contact Riemannian structure $(\Phi, \xi, \eta, g)$.

A manifold of dimension ' $n$ ' with Riemannian metric ' $g$ ' admitting a tensor field ' $\Phi$ ' of type $(1,1)$, a vector field ' $\xi$ ' and a 1-form ' $\eta$ ' satisfying (1.1), (1.3) along with

$$
\begin{gathered}
\left(\nabla_{X} \eta\right) Y-\left(\nabla_{Y} \eta\right) X=0 \\
\left(\nabla_{X} \nabla_{Y} \eta\right) Z=[-g(X, Z)+\eta(X) \eta(Z)] \eta(Y)+[-g(X, Y)+\eta(X) \eta(Y)] \eta(Z) \\
\nabla_{X} \xi=\Phi^{2} X=X-\eta(X) \xi
\end{gathered}
$$

is called a para Kenmotsu manifold or briefly $p$-Kenmotsu manifold [4]. This paper deals with a type of $p$-Kenmotsu manifold in which

$$
R(\xi, X) \cdot C=0
$$


where $C$ is the conformal curvature tensor of the manifold and $R$ is the Riemannian curvature.

Let $\left(M_{n}, g\right)$ be an $n$-dimensional Riemannian manifold admitting a tensor field ' $\Phi$ ' of type $(1,1)$, a vector field ' $\xi$ ' and a 1-form ' $\eta$ ' satisfying

$$
\left(\nabla_{X} \eta\right) Y=g(X, Y)-\eta(X) \eta(Y)
$$

$$
g(X, \xi)=\eta(X) \text { and }\left(\nabla_{X} \eta\right) Y=\varphi(\bar{X}, Y), \text { where } \varphi \text { is an associate of } \Phi
$$

is called a special $p$-Kenmotsu manifold or briefly $s p$-Kenmotsu manifold [4].

It is known that [4] in a $p$-Kenmotsu manifold the following relations hold:

$$
\begin{gathered}
S(X, \xi)=-(n-1) \eta(X) \\
g[R(X, Y) Z, \xi]=\eta[R(X, Y, Z)]=g(X, Z) \eta(Y)-g(Y, Z) \eta(X) \\
R(X, \xi)=-1 \\
R(X, \xi, \xi)=-X+\eta(X) \xi \\
R(X, \xi, X)=\xi \\
R(\xi, X, \xi)=X \\
R(X, Y, \xi)=\eta(X) Y-\eta(Y) X ; \text { when } X \text { is orthogonal to } \xi
\end{gathered}
$$

where $S$ is the Ricci tensor and $R$ is the Riemannian curvature.

Moreover, it is also known that if a $p$-Kenmotsu manifold is projectively flat then it is an Einstein manifold and the scalar curvature has a negative constant value $-n(n-1)$. Especially, if a $p$-Kenmotsu manifold is of constant curvature, the scalar curvature has a negative constant value $-n(n-1)[4]$. In this case,

$$
S(Y, Z)=-(n-1) g(Y, Z)
$$

and also

$$
R(X, Y, Z, P)=\frac{1}{(n-1)}[S(Y, Z) g(X, P)-S(X, Z) g(Y, P)]
$$

The above results will be used in the next section. 


\section{2. $p$-Kenmotsu Manifold Satisfying $R(\xi, X) \cdot C=0$}

The conformal curvature tensor $C$ is given by

$$
\begin{aligned}
C(X, Y) Z=R(X, Y) Z-\frac{1}{n-2}[g(Y, Z) Q X-g(X, Z) Q Y+S(Y, Z) X-S(X, Z) Y] \\
+\frac{r}{(n-1)(n-2)}[g(Y, Z) X-g(X, Z) Y], \quad \text { (2.1) }
\end{aligned}
$$

where $S$ is the Ricci tensor, $r$ is the scalar curvature and $Q$ is the symmetric endomorphism of the tangent space at each point corresponding to the Ricci tensor ' $S^{\prime}[5]$ i.e.,

$$
g(Q X, Y)=S(X, Y)
$$

Then

$$
\begin{aligned}
\eta(C(X, Y) Z)= & g(C(X, Y) Z, \xi) \\
=\frac{1}{n-2}[ & \left(\frac{r}{n-1}+1\right)(g(Y, Z) \eta(X)-g(X, Z) \eta(Y)) \\
& \quad-(S(Y, Z) \eta(X)-S(X, Z) \eta(Y))] .
\end{aligned}
$$

Putting $Z=\xi$ in $(2.3)$, we get

$$
\eta(C(X, Y) \xi)=0 .
$$

Again putting $X=\xi$ in (2.3), we get

$$
\begin{aligned}
& \eta(C(\xi, Y) Z)= \\
& \quad \frac{1}{n-2}\left[\left(\frac{r}{n-1}+1\right) g(Y, Z)-S(Y, Z)-\left(\frac{r}{n-1}+n\right) \eta(Y) \eta(Z)\right] .
\end{aligned}
$$

Now

$$
\begin{aligned}
(R(\xi, X) \cdot C)(U, V) W= & R(\xi, X) C(U, V) W-C(R(\xi, X) U, V) W \\
& -C(U, R(\xi, X) V) W-C(U, V) R(\xi, X) W
\end{aligned}
$$

Using (1.9), we get

$$
\begin{gathered}
R(\xi, X) C(U, V) W-C(R(\xi, X) U, V) W-C(U, R(\xi, X) V) W \\
-C(U, V) R(\xi, X) W=0 .
\end{gathered}
$$

Therefore

$$
\begin{aligned}
& g(R(\xi, X) C(U, V) W, \xi)-g(C(R(\xi, X) U, V) W, \xi) \\
& \quad-g(C(U, R(\xi, X) V) W, \xi)-g(C(U, V) R(\xi, X) W, \xi)=0
\end{aligned}
$$


From this, it follows that

$$
\begin{aligned}
& C(U, V, W, X)-\eta(X) \eta(C(U, V) W)+\eta(U) \eta(C(X, V) W)+\eta(V) \eta(C(U, X) W) \\
& \quad+\eta(W) \eta(C(U, V) X)-g(X, U) \eta(C(\xi, V) W)-g(X, V) \eta(C(U, \xi) W) \\
& \quad-g(X, W) \eta(C(U, V) \xi)=0
\end{aligned}
$$

where

$$
C(U, V, W, X)=g(C(U, V) W, X) .
$$

Putting $X=U$ in (2.8), we get

$$
\begin{gathered}
C(U, V, W, U)+\eta(V) \eta(C(U, U) W)+\eta(W) \eta(C(U, V) U)-g(U, U) \eta(C(\xi, V) W) \\
-g(U, V) \eta(C(U, \xi) W)-g(U, W) \eta(C(U, V) \xi)=0 .
\end{gathered}
$$

Let $\left\{e_{i}\right\}, i=1,2, \cdots n$ be an orthogonal basis of the tangent space at any point. Then the sum $1 \leq i \leq n$ of the relation (2.9) for $U=e_{i}$ gives

$$
\eta(C(\xi, V) W)=0 .
$$

By using (2.4), we have from (2.8)

$$
\begin{gathered}
C(U, V, W, X)-\eta(X) \eta(C(U, V) W)+\eta(U) \eta(C(X, V) W)+\eta(V) \eta(C(U, X) W) \\
+\eta(W) \eta(C(U, V) X)-g(X, U) \eta(C(\xi, V) W)-g(X, V) \eta(C(U, \xi) W)=0 .
\end{gathered}
$$

In virtue of (2.5) and (2.10), we have

$$
S(Y, Z)=\left(\frac{r}{n-1}+1\right) g(Y, Z)-\left(\frac{r}{n-1}+n\right) \eta(Y) \eta(Z) .
$$

Using (2.3), (2.4), (2.10) and (2.12), the relation (2.11) reduces to

$$
C(U, V, W, X)=0 \text {. }
$$

From (2.13) it follows that

$$
C(U, V) W=0 .
$$

which proves that the $p$-Kenmotsu manifold is conformally flat and hence it is of constant curvature.

Then from (1.19) and (1.20) we have

$$
\text { ' } R(X, Y, Z, P)=g(X, Z) g(Y, P)-g(Y, Z) g(X, P)
$$


Also from (1.19) and (1.5) we have

$$
S(\Phi X, \Phi Y)=-(n-1)[g(X, Y)-\eta(X) \eta(Y)]
$$

On contraction (2.16) with covariant tensor $\varphi(X, Y)=g(\bar{X}, Y)$ and hence we find

$$
\varphi(\bar{X}, Y)=g(X, Y)-\eta(X) \eta(Y)
$$

that is, the manifold is an $s p$-Kemotsu one. Thus, we can state the following theorem.

Theorem: If a $p$-Kenmotsu manifold $\left(M_{n}, g\right)$ is conformally flat, the manifold is an $s p$-Kenmotsu one.

Acknowledgements: The authors acknowledge Prof. Kalpana of Banaras Hindu University, Dr. B. Satyanarayana of Nagarjuna University and Dr. A. Kameswara Rao of GVP College of Engineering for Women for their valuable suggestions in preparation of the manuscript.

\section{References}

[1] I. Sato, On a structure similar to the almost contact structure, Tensor (N.S.), 30 (1976), 219-224.(doi)

[2] T. Adati and K. Matsumoto, On conformally recurrent and conformally symmetric P-Sasakian manifolds, TRU Math., 13 (1977), 25-32.

[3] K. Kenmotsu, A class of almost contact Riemannian manifolds, Tohoku Math. Journal, 24 (1972), 93-103, doi:10.2748/tmj/1178241594.

[4] B. B. Sinha and K. L. Sai Prasad, A class of almost para contact metric Manifold, Bulletin of the Calcutta Mathematical Society, 87 (1995), 307312.

[5] R.L. Bishop and S. I. Goldberg, On conformally flat spaces with commuting curvature and Ricci transformations, Canad. J. Math., 14, No.5 (1972), 799-804, doi:10.4153/CJM-1972-077-6.

[6] M. C. Chaki and B. Gupta, On conformally symmetric spaces, Indian J. of Math., 5 (1963), 113-122. 
[7] U.C. De and N. Guha, On a type of P-Sasakian manifold, Istambul Univ. Fen Fak. Mat. Der., 51 (1992), 35-39.

[8] M. Tarafdar and A. Mayra, On P-Sasakian manifold, Istambul Univ. Fen Fak. Mat. Der., 53 (1994), 73-76.

[9] Cihan Ozgur, On Kenmotsu manifolds satisfying certain pseudosymmetry conditions, World Applied Sciences Journal, 1, No.2 (2006), 144-149.

[10] K.L.Sai Prasad, Certain classes of almost contact Riemannian Manifolds, International Mathematical Forum, 16, No.4 (2009), 773-778.

[11] T. Satyanarayana and K.L. Sai Prasad, On a type of Para Kenmotsu Manifold, Pure Mathematical Sciences, 2, No.4 (2013), 165 - 170. 
